Annals of Glaciology 191994

(C) International Glaciological Society

\title{
Bent icicles and spikes
}

\author{
N. Maeno, \\ Institule of Low Temperature Science, Hokkaido University, Sapporo 060, Japan \\ L. MAKKONEN, \\ Laboratory of Structural Engineering, Technical Research Center of Finland, 02150 Espoo, Finland \\ T. TAKAHASHI \\ Hokkaido University of Education, Sapporo 002, Japan
}

\begin{abstract}
Formation mechanisms of bent icicles and thin spikes were studied by observations of natural icicles and by artificially growing icicles in a cold laboratory, and the results are discussed with reference to the general growth process of icicles. It is shown that icicles can be bent by the displacement of their roots, change in water supply rates, and wind. Spikes were observed to grow by the freezing of a water column captured at the centre of icicles after their length and diameter growth stopped.
\end{abstract}

\section{INTRODUGTION}

The physical process of icicle growth has been investigated extensively by Geer (1981), Maeno and Takahashi (1984a, b), Makkonen (1988) and Maeno and others (in press). However, there remain several important phenomena, which were noted in their studies but have not been surveyed in detail. Maeno and Takahashi (1984b) tried to clarify mechanisms for the bending of icicles and for the formation of thin spikes often found on the surface of an icicle. This paper describes further studies on bent icicles and spikes.

\section{BENT IGICLES}

Bent icicles are often observed to grow from roofs and trees (Fig. 1). Maeno and Takahashi (1984b) showed that at least three factors can result in the formation of bent icicles. The first is any inclination of roots of icicles, e.g. by the creeping deformation of ice/snow on a roof edge or by bending of a tree branch from which icicles are growing. Such bent icicles are often observed to grow from trees exposed to spray and waves of river, lake or sea water. The second is the change of water supply conditions during icicle growth. A decrease in water supply rate by any meteorological cause will lead to a variation of waterflow pattern; a single flow path on the icicle wall will result in a bent icicle, and two flow paths, forked icicles. In our icicle growth experiments the above two types of bent icicles could easily be produced.

The third factor to bend icicles is the effect of wind. To study various effects of wind on icicle formation, an icicle growth device was constructed in a cold laboratory, the temperature of which was varied between 0 and $-30^{\circ} \mathrm{C}$. The device was similar to that used by Maeno and Takahashi (1984a) and Maeno and others (in press), in which icicles could be grown from a horizontal metal bar wrapped by wet gauze, to which pure water just above the melting temperature of ice was supplied at a constant rate between 0 and $50 \mathrm{mg} \mathrm{s}^{-1}$. Wind speed could be varied between 0 and $6.0 \mathrm{~m} \mathrm{~s}^{-1}$. During the growth process the icicle's length and diameter were measured, and relative humidity, maintained at about $80 \%$, was also monitored.

It was found that wind affects icicle growth in two ways: it increases the heat exchange and changes the water flow at the wall and tip. In our experiments the latter effect was more important than the former; it is observed that the horizontal cross-section of an icicle grown in windy conditions becomes "anti-streamlined"

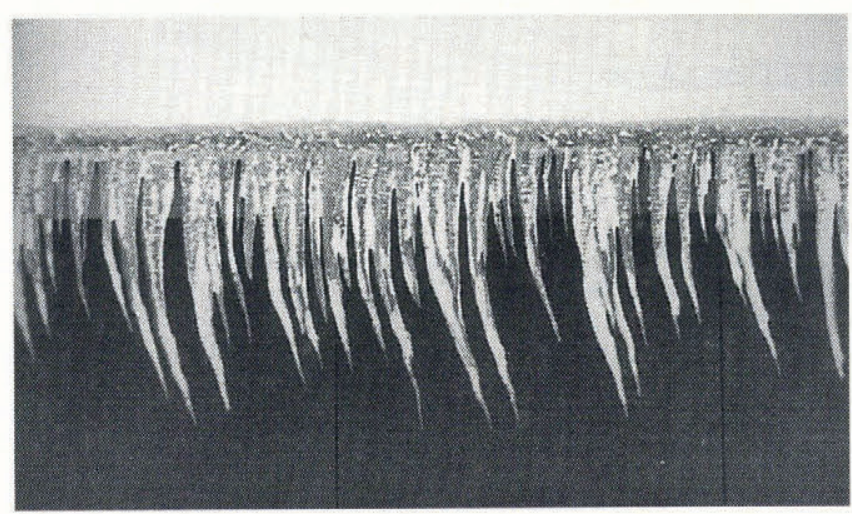

Fig. 1. Natural bent icicles growing from a roof. All the icicles are bent toward the righhand side by wind. The horizontal width of the photograph is $2.5 \mathrm{~m}$. 


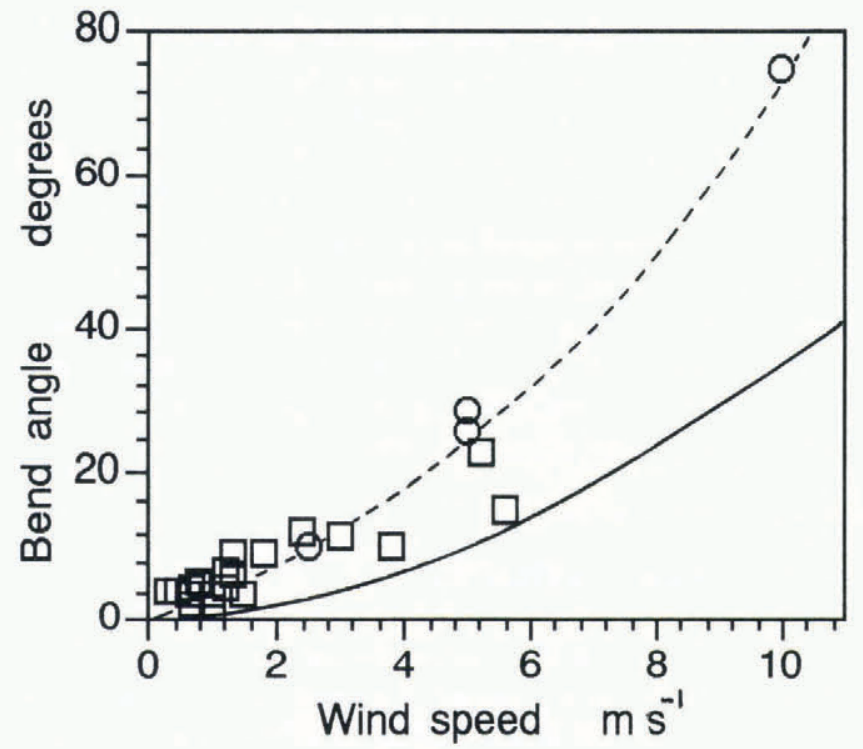

Fig. 2. Measured bend angle versus wind speed. $\square$ : present results (temperature around $-9^{\circ} \mathrm{C}$ and water-supply rate around $\left.10 \mathrm{mg} \mathrm{s}^{-1}\right)$. $\mathrm{O}$ : data from Makkonen and Fujii (1993). The dashed and solid curves refer respectively to the empirical $\left(\phi=0.684 \bar{U}^{2}+0.14 \bar{U}+\right.$ 4.0 degrees, $\bar{U}$ in $\mathrm{ms}^{-1}$ and calculated (Equation (1)) bend angles.

with the sharper end windward, the lee end becoming blunter and thicker due to the more active growth of ice. The faster growth is attributed to the wind-driven film flow of water, which is cooled effectively by the wind, towards leeward at the wall, leading to anti-streamlined or flat icicles. Microscope observation of thin crosssections of these icicles under polarized light showed that crystal grains are generally much smaller and contain more tiny air bubbles at the lee, suggesting faster freezing of water.

When wind was started after some growth of an icicle, mostly $20 \mathrm{~cm}$, the icicle began to bend sharply. The angle of bend, measured from vertical, was found to increase with the wind speed in the range studied; in the measurement the air temperature and water supply rate were maintained at about $-9^{\circ} \mathrm{C}$ and $10 \mathrm{mg} \mathrm{s}^{-1}$ respectively. Figure 2 gives the measured bend angle plotted against wind speed together with the result by Makkonen and Fujii (1993), who grew icicles on a horizontal wire in an icing wind tunnel. In the figure the dashed line is a curve fitted to the measured data.

The general increase in bend angle with wind speed is reasonable because an aerodynamic force acts on the pendant drop at the tip where the icicle is growing lengthwise. If we assume that a spherical pendant drop (radius $r$ ) is in a mechanical balance between gravity and drag force due to a steady wind (horizontal speed $U$ ), a force balance equation leads approximately to

$$
\tan \phi=\left(3 \rho_{\mathrm{a}} C_{\mathrm{D}} U^{2}\right) /\left(8 \rho_{\mathrm{w}} g r\right)
$$

where $\phi$ is the bend angle, $\rho_{\mathrm{a}}$ and $\rho_{\mathrm{w}}$ are the densities of air and water respectively, $C_{\mathrm{D}}$ is the drag coefficient, and $g$ is the acceleration of gravity. According to the observation of Maeno and others (in press), $r$ increases only slightly with water supply rate and is kept constant at $2.44 \mathrm{~mm}$ in average irrespective of temperature and the Reynolds number $\left(\operatorname{Re}=2 \rho_{\mathrm{a}} r U / \eta, \eta\right.$ : dynamic viscosity of air). Re is roughly in the range of 350 to 3500 in the experimental conditions, when $U=1-10 \mathrm{~m} \mathrm{~s}^{-1}$. The drag coefficient is a function of Reynolds number and can be empirically expressed as follows (Morsi and Alexander, 1972):

$$
C_{\mathrm{D}}=98.33 \mathrm{Re}^{-1}-2778 \mathrm{Re}^{-2}+0.3644
$$

for $100<\operatorname{Re}<1000$, and

$$
C_{\mathrm{D}}=148.62 \mathrm{Re}^{-1}-4.75 \times 10^{4} \mathrm{Re}^{-2}+0.357
$$

for $1000<\mathrm{Re}<5000$. The solid line in Figure 2 gives the bend angle of an icicle at various wind speeds calculated by Equation (1) together with Equations (2) and (3). Equation (1) can explain only the general tendency of increase in the measured bend angle with wind speed. The magnitudes of the measured angles are much larger than those calculated, implying that the bend of icicles is caused by wind action but not only through the simple mechanical aerodynamical force acting on a pendant drop given by Equation (1).

In windy conditions, part of wind-driven flow of effectively cooled water freezes at the leeward wall forming an anti-streamlined icicle, as stated above, and the rest finally enters into a pendant drop preferentially at the lee, which leads to faster growth there and consequently allows the icicle to bend. At this stage of study, however, the detailed flowing and freezing process cannot be formulated reasonably, because more quantitative information is needed on several important processes involved such as the air flow around an icicle which controls the water flow and heat exchange, the viscous flow of a thin, roughly $100 \mu \mathrm{m}$, water film in which radial (horizontal) temperature and velocity distributions are set up, and so on.

\section{FORMATION MECHANISM OF SPIKES}

The photographs in Figure 3 show natural icicles with spikes. Spikes are thin ice needles typically $1 \mathrm{~mm}$ in diameter and 5 to $20 \mathrm{~mm}$ in length. Some, however, are much thicker and shorter. Under the polarized light, microscope observations were made of spikes produced on natural and artificially formed icicles. The spikes were found to be composed of two or a few single crystals. The growth direction of spikes is random and shows no systematic relation to that of main icicle growth. Some icicles have several spikes and some none, depending on how their growth stops, as explained below.

It was found from observations of natural icicles and those formed in a cold laboratory that spikes are not a peculiar or unusual pheomenon. In our icicle formation experiment most icicles were observed to produce spikes after the cessation of their growth of length and diameter and the subsequent slow freezing of a water column captured at the centre of each icicle. These spike production events sometimes took place a few hours after the overall growth of an icicle depending on its growth history, size and surrounding temperature. 


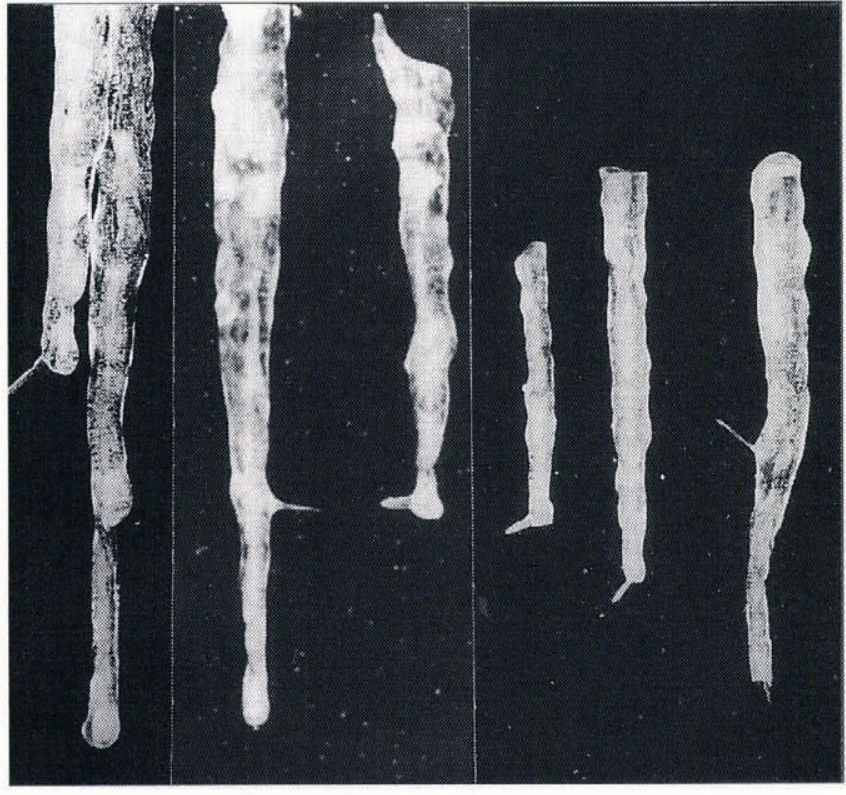

Fig. 3. Natural icicles with spikes. The vertical length of the photograph is $180 \mathrm{~mm}$.

As shown by Maeno and Takahashi (1984a), Makkonen (1988) and Maeno and others (in press), an icicle grows by a simultaneous freezing of water at the tip and wall, the growth being maintained by a balance between the supply rate and freezing rate of water. When the balance is destroyed and dripping of water drops at the tip ceases, the pendant drop begins to freeze from outside forming a thin ice shell. As freezing proceeds the pressure inside increases steadily. Since the pendant drop is connected to a water cylinder, $5 \mathrm{~mm}$ in diameter and a few $\mathrm{cm}$ in length, in the central part of an icicle, its subsequent freezing produces a high internal pressure which squeezes out unfrozen water from small holes and cracks. Water exiting from such holes and cracks rapidly freezes to form spikes. Thus various forms of spikes can be produced in various directions depending on the mode of water squeezing and freezing.

When an icicle stops growing, the water film on the wall also begins to freeze from the outer surface which is in contact with the cold air. In this case, only very fine needle spikes may be formed since the volume of confined water is small so that the pressure produced is also small.

Spikes are so thin that they easily disappear by melting or sublimation. This may be the reason why spikes have not been frequently noticed, although they should form in every icicle when unfrozen water is confined with a thin ice shell after the cessation of growth. It was noticed that spikes are more frequently formed at the tip than near the root, and in the late afternoon when most icicles stop growth by the relative decrease in the water supply rate and increase in the freezing rate.

The formation mechanism of spikes on icicles is essentially the same as the growth of tubular or columnar ice, which develops from an ice layer covering supercooled water (Dorsey, 1921; Bally, 1935; Blanchard, 1951; Hayward, 1966; Krausz and others, 1967; Tusima and Suzuki, 1972; Wascher, 1991).

\section{AGKNOWLEDGEMENTS}

The work was partly supported by Grants-in-Aid for Research in Natural Disaster, Co-operative Research and Scientific Research of the Ministry of Education, Science and Culture. The authors thank anonymous referees for their helpful comments.

\section{REFERENCES}

Bally, O. 1935. Über eine eigenartige Eiskrystallbildung. Helv. Chim. Acta, 18, $475-476$.

Blanchard, D. 1951. A verification of the Bally-Dorsey theory of spicule formation on sleet pellets. F. Meteorol., 8, 268-269.

Dorsey, H. G. 1921. Peculiar ice formations. Phys. Rev., 17, 162-164.

Geer, I. W. 1981. The not-so-ordinary icicle. Weatherwise, 34(6), 257259.

Hayward, A. T.J. 1966. Growth of ice tubes. Nature, 211, 172-173.

Krausz, A. S., B. Harron and G. G. Litvan. 1967. Tubular ice crystals. Nature, 215(5098), 271-273.

Maeno, N. and T. Takahashi. 1984a. Studies on icicles. I. General aspects of the structure and growth of an icicle. Low Temp. Sci., Ser. A 43, 125-138. [In Japanese with English summary.]

Maeno, N. and T. Takahashi. 1984b. Studies on icicles. II. Wave-forms, spikes and bent icicles. Low Temp. Sci., Ser. A 43, 139-147. [In Japanese with English summary.]

Maeno, N., L. Makkonen, K. Nishimura, K. Kosugi and T. Takahashi. In press. Growth rates of icicles. J. Glaciol.

Makkonen, L. 1988. A model of icicle growth. J. Glaciol., 34(116), 64 70.

Makkonen, L. and Y. Fujii. 1993. Spacing of icicles. Cold Reg. Sci. Technol., 21 (3), 317-322.

Morsi, S. A. and A.J. Alexander. 1972. An investigation of particle trajectories in two-phase flow system. F. Fluid Mech., 55(2), 193-208.

Tusima, K. and S. Suzuki. 1972. Columnar ice protruded to the air. Low Temp. Sci., Ser. A 30, 23-33. [In Japanese with English summary.]

Wascher, T. 1991. Generation of slanted gas-filled icicles. 7. Cryst. Growth, 110, 942-949

The accuracy of references in the text and in this list is the responsibility of the authors, to whom queries should be addressed. 